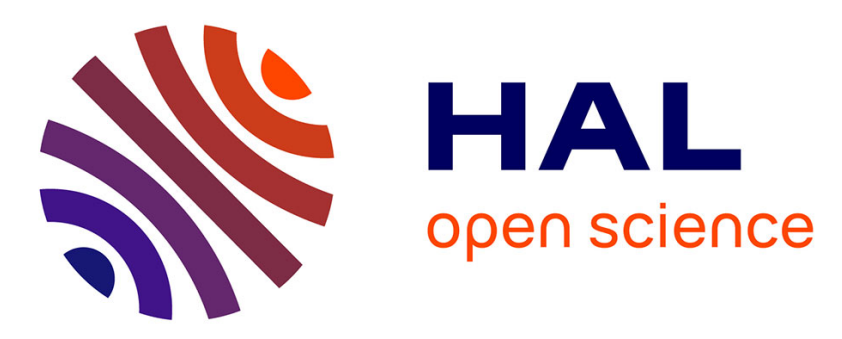

\title{
Balancing Block Copolymer Thickness over Template Density in Graphoepitaxy Approach
}

\author{
Patricia Pimenta Barros, Ahmed Gharbi, Antoine Fouquet, Sandra Bos, \\ Jérôme Hazart, Florian Delachat, Xavier Chevalier, Ian Cayrefourcq, Laurent \\ Pain, Raluca Tiron
}

\section{To cite this version:}

Patricia Pimenta Barros, Ahmed Gharbi, Antoine Fouquet, Sandra Bos, Jérôme Hazart, et al.. Balancing Block Copolymer Thickness over Template Density in Graphoepitaxy Approach. Macromolecular Materials and Engineering, 2017, 302 (11), pp.1700285. 10.1002/mame.201700285 . cea-02111234

\section{HAL Id: cea-02111234 https://hal-cea.archives-ouvertes.fr/cea-02111234}

Submitted on 25 Apr 2019

HAL is a multi-disciplinary open access archive for the deposit and dissemination of scientific research documents, whether they are published or not. The documents may come from teaching and research institutions in France or abroad, or from public or private research centers.
L'archive ouverte pluridisciplinaire HAL, est destinée au dépôt et à la diffusion de documents scientifiques de niveau recherche, publiés ou non, émanant des établissements d'enseignement et de recherche français ou étrangers, des laboratoires publics ou privés. 
Article type: Full paper

\title{
Balancing Block Copolymer Thickness over Template Density in Graphoepitaxy Approach
}

\author{
Patricia Pimenta Barros*, Ahmed Gharbi, Antoine Fouquet, Sandra Bos, Jérôme Hazart,
} Florian Delachat, Xavier Chevalier, Ian Cayrefourcq, Laurent Pain, Raluca Tiron

P. Pimenta Barros, Dr. A. Gharbi, A. Fouquet, S. Bos, Dr. J. Hazart, Dr. F. Delachat, Dr. L. Pain, Dr. R. Tiron

CEA-LETI, 17 Rue des Martyrs, 38054 Grenoble, France

E-mail : patricia.pimenta-barros@cea.fr

Dr. X. Chevalier, Dr. I. Cayrefourcq

ARKEMA FRANCE, Route Nationale 117, BP34, 64170 Lacq, France

Keywords: block copolymer; Directed Self-Assembly (DSA); graphoepitaxy; pattern density; planarization

Directed Self-Assembly (DSA) of Block Copolymers (BCP) is one of the most promising patterning solutions for sub-10 nm nodes. While significant achievements have been demonstrated in pattern fidelity for various applications (contact shrink, line patterning), some challenges still need to be overcome especially regarding the defect density reduction, in order to ensure DSA insertion in high-volume manufacturing. In particular, in the case of the graphoepitaxy approach, a remaining challenge is to solve the pattern-densities-related defect issue due to $\mathrm{BCP}$ film thickness variation inside the guiding template. In order to address this issue, a new DSA process flow called "DSA planarization" is employed for contact-hole patterning, and consists in overfilling the guiding pattern cavities with a thick BCP film, followed by a plasma etch-back step. This new approach ensures a uniform control of the final thickness of the BCP inside guiding cavities of different densities, as demonstrated herein by AFM measurement. Thus, defect-free isolated and dense patterns for both contact shrink and multiplication is simultaneously resolved. Furthermore, the simulation results of BCP self-assembly overfilling the templates demonstrate 
that BCP domains are well directed in vertical cylinders ordering inside guiding cavities, which confirms the experimental results and the viability of this approach.

\section{Introduction}

For the sub-10nm technology nodes, the semiconductor industry is facing the limits of conventional lithography to achieve narrow dimensions. Among different emerging patterning techniques, Directed Self-Assembly (DSA) of Block Copolymers (BCP) is still one of the most promising solutions due to its simplicity, its cost-effectiveness and its capability to generate high density patterns with different shapes (contact, line and space). ${ }^{[1-3]}$ Moreover, several demonstrations of DSA have already been proposed in literature for advanced devices patterning, such as FinFet-like transistor with a $40 \mathrm{~nm}$ fin pitch $^{[4,5]}$ or contact/via patterning in $22 \mathrm{~nm}$ SRAM devices. ${ }^{[6,7]}$ In order to control domain registration, there are two main approaches for DSA: chemoepitaxy and graphoepitaxy. While chemoepitaxy directs the BCP self-assembly by locally changing the surface properties of the substrate to create chemical guiding patterns or prepatterns, ${ }^{[8,9]}$ graphoepitaxy relies on directing the self-assembly of BCPs inside topographical guiding patterns by physical confinement. ${ }^{[10,11]}$ The latter approach, which is extensively used for contact hole patterning, has shown a major issue concerning the defectivity level dependence on the guiding pattern density. ${ }^{[12,13]}$ This issue presents a serious concern for DSA integration as different densities can exist in an interconnect level of an IC circuit. ${ }^{[12-14]}$ Indeed, in the graphoepitaxy approach, the guiding patterns are filled by the BCP film which is deposited by spin-coating. Several authors including ourselves have observed a BCP thickness variation depending on the guiding patterns dimension and density ${ }^{[12-15]}$ resulting in a locally thicker film for isolated patterns and matrix edges, and in a thinner polymer film on the top of dense features. Hence, the BCP thickness variation occurring on the same processed wafer would lead to 
defectivity issues after BCP self-assembly and therefore would induce missing contacts after $\mathrm{BCP}$ pattern transfer by plasma etching.

In the literature, some solutions were investigated in order to overcome the DSA process dependence on the templates density. For instance, retentions cavities, judiciously sized and placed in a given layout, could be a way to improve the filling uniformity of $\mathrm{BCP}$ inside guiding patterns. ${ }^{[16]}$ The retentions cavities (also called "assist features") printed on the wafer should have a targeted opening area to absorb the $\mathrm{BCP}$ material in excess around a low-density pre-pattern. In addition, they should be printed at low dimension, not commensurable with the $\mathrm{BCP}$ chain length, and thus avoiding the formation of unwanted DSA holes. H. Yi et al. ${ }^{[17]}$ and J. Bekaert et al. ${ }^{[18]}$ have recently reported first experimental demonstrations of this approach. Their work showed that the influence of pre-pattern density on DSA defectivity could be solved using printed sub-DSAresolution assist features (SDRAF) in the graphoepitaxy process. However, such aggressive retention cavities (Critical Dimension $<40 \mathrm{~nm}$ ) will exhibit a very narrow process window when generated by conventional i-193nm lithography. The use of i-193nm lithography beyond its conventional limits could lead to important process variation and thus randomly generate unwanted pre-patterns contacts. Furthermore, this approach will add more complexity to the final design layout.

In this paper, in order to balance the BCP film thickness over template density, we propose a new and simple graphoepitaxy process flow called "DSA planarization" for contact hole patterning. The DSA planarization process enables to make the BCP film thickness uniform inside the cavities regardless of their densities, by means of appropriate BCP spin-coating and subsequent etch-back step. ${ }^{[19-21]}$ In this work, simulations of BCP self-assembly overfilling the guiding patterns are performed in order to understand and predict the behavior of BCP self-assembly during the DSA 
planarization process, i.e. when the BCP film overfills the guiding patterns. Then, we experimentally demonstrate that the "DSA planarization" approach allows the enhancement of DSA performances, more precisely by removing the defectivity related to pre-pattern density variation. For this purpose, the Hole Open Yield (HOY) of contact-hole patterns is measured using top-view SEM inspection. In parallel, the BCP film thickness inside templates is monitored using AFM.

\section{Results and Discussion}

In this work, we have investigated the impact of guiding patterns density on DSA graphoepitaxy approach, where the BCP film is deposited by spin-coating onto a patterned wafer. The filling of pattern templates during the spin-coating of a polymer film depends on the spin-coating conditions and also on the pattern opening rate on the wafer. ${ }^{[22,23]}$ Consequently, for a given $\mathrm{BCP}$ spin-coating condition, it is expected to obtain different BCP filling of the templates depending on their densities in a standard graphoepitaxy approach (figure 1. (a)). Indeed, more material is needed to locally fill a dense pattern area compared to a sparse pattern area, which results in the underfilling of dense patterns and overfilling of isolated patterns patterns, respectively. This pattern density effect has been observed experimentally in previous works. ${ }^{[12-14]}$

Overcoming the density dependence issue of DSA graphoepitaxy process involves filling all guiding patterns of different densities with the same BCP thickness. To achieve this goal, we propose the process flow called "DSA planarization" which enables to planarize the patterned surface with the BCP film by means of appropriate BCP spin-coating and etch-back steps as depicted in figure 1. (b). First, the BCP film is spin-coated onto the wafer with high film thickness in order to overfill the cavities of all guiding patterns of different opening densities and to get a surface as flat as possible. Afterwards, the BCP is self-assembled by thermal annealing. The 
annealing temperature is optimized depending on the thickness of BCP film deposited onto the wafer. Here, a PS- $b$-PMMA BCP system with a cylindrical morphology and a natural period $\left(\mathrm{L}_{0}\right)$ of $35 \mathrm{~nm}$ has been used in order to shrink the contact-holes pattern. After the BCP self-assembly step, the PMMA cylinders are expected to be in a vertical ordering in the depth of the BCP film inside the guiding pattern cavities, due to the physical confinement and the surface affinity of the guiding patterns. However, at the top-surface of the wafer, i.e. outside the guiding patterns cavities, the BCP film is not physically constrained by the guiding patterns, which could generate in-plane PMMA cylinders that need to be removed. Hence, a plasma etch-back step is performed with $\mathrm{O}_{2}$-based chemistry in order to keep only the BCP inside the guiding patterns cavities. For better etching control, an inorganic sublayer (SiARC in our case) is used to act as an etch-stop layer during the etch-back step. This permits to detect the etching end of BCP in excess outside the pre-patterns cavities. It is important to note that the self-assembly annealing should be performed just after the BCP spin-coating and before the plasma etch-back step. Indeed, the ultraviolet radiation during plasma etch-back processing may induce the damage of the PS and PMMA blocks (via chain scission and covalent bonds cleavage) ${ }^{[24,25]}$ which prevents the selfassembly of BCP to take place when subsequent thermal annealing is carried out.

The planarization of topographical substrate by spin-coating is already known and widely used in microelectronics, ${ }^{[22,23]}$ but it has never been performed with a BCP film that is able to generate self-aligned nanodomains. The challenge of the DSA planarization process flow is to control the BCP thickness homogeneity on the wafer regardless of the guiding template size and densities and, at the same time, to ensure an efficient and defect-less self-assembly of the BCP film for contacthole patterning. 
Next, three-dimensional (3D) simulations have been performed in order to understand and predict the behavior of $\mathrm{BCP}$ self-assembly during the $\mathrm{DSA}$ planarization process, i.e. when the $\mathrm{BCP}$ film overfills the guiding patterns. The purpose of the simulation is also to determine if there are any interactions between the nanodomains formed outside and inside the cavities, and if so to quantify the interaction length.

Herein, a PS- $b$-PMMA BCP with an intrinsic period $\left(\mathrm{L}_{0}\right)$ of $35 \mathrm{~nm}$ was used for the simulation. The simulated guiding patterns were defined with a Critical Dimension (CD) of $65 \mathrm{~nm}$, a neutral affinity at the bottom of pre-patterns, a PMMA affinity on the pre-patterns sidewalls and the topsurface, in order to correspond to our experimental conditions. The BCP self-assembly model is based on a phase-field method, more precisely using a Cahn-Hilliard equation with Ohta-Kawasaki energy functional. ${ }^{[26]}$ To mimic the high $\mathrm{BCP}$ thickness over the guiding patterns cavities, a $\mathrm{BCP}$ bulk of variable thickness was added to the simulation domain on the top of the cavity. To scale the equation's parameters to experimental data, we used scaling laws derived by Choksi. ${ }^{[27,28]}$ The segregation strength $(\chi N)$ was fixed to 20 according to BCP's properties and the iterations was stopped when the system reached equilibrium. Figure 2 reports the simulation results of BCP selfassembly as a function of the BCP over-thickness height referred as T. For the whole range of $\mathrm{T}$ between 0 up to $1,75 \mathrm{~L}_{0}$, we observe that the PMMA cylinder stays perfectly straight inside the guiding pattern cavity, even if additional in-plane cylinders are formed in the upper part of BCP film outside the cavity. This result is explained by the fact that the BCP film outside the prepatterns cavities is not constrained and reacts as it was deposited on free-surface wafer (i.e without guiding patterns); whereas in the depth of the BCP film, the guiding patterns force the BCP's selfassembly to be in vertical cylinder ordering. The simulation results also suggest that depending on BCP over-thickness height $(\mathrm{T})$ the top of the vertical PMMA cylinder is not always reaching the 
top of the guiding cavity. Indeed, the number of in-plane cylinder layers is increasing with the film thickness and depending on the commensurability of $\mathrm{BCP}$ over-thickness height $(\mathrm{T})$ with $\mathrm{L}_{0}$. Moreover, this morphology on the top is pushing more or less the in-depth vertical cylinder inside the cavity. For instance, for a BCP over-thickness equal to $0.5^{*} \mathrm{~L}_{0}$, a semi sphere is formed on top of the cavity and prevents the in-depth vertical cylinder from reaching the top of cavity. However, for a $\mathrm{BCP}$ over-thickness equal to $1.25^{*} \mathrm{~L}_{0}$, the in-depth vertical cylinder reaches the top of the cavity without being impacted by the in-plane cylinders formed above. According to our simulation, the distance (d) between the top of the cavity and the top of vertical PMMA cylinder is $17 \mathrm{~nm}$ at maximum, i.e. $\mathrm{L}_{0} / 2$.

These simulation results are very encouraging for the use of DSA planarization approach as they predict that $\mathrm{BCP}$ is well directed in vertical cylinder ordering inside guiding patterns. The in-plane PMMA cylinders predicted on the top-surface are considered as defects for contact-hole application, but this can be overcome using an etch-back step.

Moreover, these simulation results are consistent with the experimental observation as reported in figure 3. Indeed, the cross-sectional SEM image of a 200nm-thick BCP film overfilling the guiding patterns after the BCP's self-assembly step (figure 3. (b)) shows a multi-layer of horizontal cylinders with a hexagonal mesh ordering above the guiding pattern cavity (area $\mathrm{A}_{0}$ ). The SEM image confirms that BCP follows an "in-plane" ordering and behaves as it was self-assembled on a free-surface (i.e. without guiding patterns). By examining the area $A_{2}$ (inside the cavities), we can observe vertical holes, which confirm the simulation predictions. Nevertheless, due to the low contrast between the PS and the PMMA phase during SEM observation, it is difficult to verify if there is an unstable $\mathrm{BCP}$ ordering at the beginning of the cavity (area $\mathrm{A}_{1}$ ). It is not possible to measure experimentally the distance between the last horizontal cylinder from the upper $\mathrm{BCP}$ layer 
and the vertical cylinder that begins inside the cavity. According to our simulation results, this distance (d) is limited to $17 \mathrm{~nm}$ at the most.

As horizontal cylinders induce defects for contact-hole applications, a well-controlled etch-back step is mandatory to reach the in-depth vertical cylinder with precision. For this purpose, the etchback step was monitored using an Optical Emission Spectroscopy (OES) system installed on the etching chamber. The OES technique permits to follow the evolution of the light emitted by the excited species produced during the plasma etching, in the UV/Visible (200nm-850nm) range. ${ }^{[29]}$ The most significant by-products involved in the plasma etching of the $\mathrm{BCP}$ film with $\mathrm{O}_{2}$-based chemistry are derived from carbon, hydrogen and oxygen atoms. Herein, the wavelengths, corresponding to the emission of CO species $(\lambda=483 \mathrm{~nm}), \mathrm{H}$ species $(\lambda=656 \mathrm{~nm})$ and $\mathrm{OH}$ species $(\lambda=309 \mathrm{~nm}$ ) were used to monitor the etch-back step (figure 4). The drastic decrease of these wavelength signals corresponds to the end of the upper BCP layer etching and it shows that the inorganic etch-stop layer at the top surface of the cavity is reached. From this point, the BCP film inside the cavity will start to be etched. Thus, the etch-back process can be divided into two regions: a main-etch $(\mathrm{ME})$ region corresponding to the etching of the $\mathrm{BCP}$ film above the cavity, and an over-etch (OE) region corresponding to the etching of the first $17 \mathrm{~nm}-\mathrm{BCP}$ film at the beginning of the cavity. An OE of $8 \mathrm{~s}$ was necessary to etch the first $17 \mathrm{~nm}$-BCP film according to the etch-rate of the $\mathrm{O}_{2}$-based plasma process. An automatic endpoint detection was performed to switch properly from the ME to the OE step.

Besides, on the ME region shown in figure 4, one can observe a periodic oscillation of the wavelengths intensity. These oscillations indicate a periodic variation of the $\mathrm{OH}, \mathrm{H}$ and $\mathrm{CO}$ species during plasma etching, which could correspond to the alternative etching of the PS and PMMA phase above the cavity. Indeed, according to the SEM cross-section image in figure 3(b), the BCP 
film above the cavity is composed of 7 layers of in-plane PMMA cylinders. This morphological observation is consistent with the periodic oscillations composed of 7 pics. Moreover, the PMMA phase is expected to release more $\mathrm{OH}$ and $\mathrm{CO}$ species than the PS during plasma etching, as PMMA contains $\mathrm{O}$ atoms on its backbone unlike PS. For this reason, the 7 pics of the periodic oscillations observed in the ME region were attributed to the etching of in-plane PMMA cylinders layers. According to our experimental results, the 309 and 656 wavelengths give higher intensity contrast between the PMMA and PS etching. These results on the OES signals suggest that the etching of the upper BCP film may be performed and monitored layer by layer. Moreover, the periodic oscillations inform us on how BCP is self-assembled in the upper layer. Thus, the OES signals are also an indirect way to characterize the $\mathrm{BCP}$ self-assembly through the wafer.

Figure 4 shows the top-view SEM image taken at different etching times: before etching ( $\left.\mathrm{t}_{0}\right)$, after ME step $\left(t_{1}\right)$, and after ME + OE steps $\left(t_{2}\right)$. The top-view SEM images corresponding to $t_{0}$ show in-plane PMMA cylinders on the wafer top surface, confirming the simulations results and crosssectional observations. After ME at $t_{1}$, we can observe some missing holes on the top-view SEM images, indicating that we did not reach the in-depth vertical cylinder of BCP film. This image also suggests that there is an unstable $\mathrm{BCP}$ ordering varying from one cavity to another. Therefore, the OE step is then mandatory to etch the non-desired BCP layer and reach the in-depth vertical cylinders. The top-view SEM images taken after ME + OE steps corresponding to $t_{2}$ in figure 4 confirm that the in-depth vertical ordering can be reached as no missing contacts are observed at this step.

Herein, the etch-back steps were performed in an Inductive Coupled Plasma (ICP) etcher using the same $\mathrm{O}_{2}$-based etching process for both the $\mathrm{ME}$ and $\mathrm{OE}$ steps. The plasma conditions have been optimized in order to conserve the pattern-hole dimension and enough remaining $\mathrm{BCP}$ thickness 
for transfer into underlayers. In particular, the $\mathrm{O}_{2}$ amount in the gas mixture was carefully adjusted to minimize the isotropic component of the etching that could otherwise lead to an increase of hole dimension. Moreover, a low etch-rate is recommended for better etching control, especially for the OE step. Thus, the plasma etching steps were performed at a low input power and low RF bias for respectively minimizing the etch-rate and not damaging the hole-patterns. As perspectives, it will be interesting to study the possibility to use two different etching processes for the ME and $\mathrm{OE}$ steps respectively. Indeed, the etching of the upper-BCP layer outside the cavity needs a low PMMA/PS selectivity in order to etch the two phases at the same time. Different PS and PMMA etch rates during the ME step could lead to the transfer of PS patterns coming from the BCP overfilling above the cavity, which would generate a stacking of in-plane and vertical cylinders. ${ }^{[30,}$ ${ }^{31]}$ Such complex structures are not wanted for the contact-shrink and multiplication applications presented in this paper. Therefore a low PMMA/PS selectivity (inferior to 2) is recommended for the ME step. However, an isotropic etching has to be avoided or very limited during the OE step in order to preserve the hole dimensions. A higher etching selectivity during the OE step may guarantee better control of pattern-hole dimension, for example. Otherwise, different etching strategies could be envisaged for the etch-back steps of DSA planarization process depending on the targeted applications (contact or line/space) and the block copolymer system employed. Thus, DSA planarization approach could directly be applied to high-chi BCP materials that are the nextgeneration BCPs for DSA lithography. ${ }^{[32,33]}$

The simulations and experimental results have shown that DSA planarization process flow can ensure a good self-assembly of the PS- $b$-PMMA BCP film inside the guiding patterns for contacthole patterning. In the following, the DSA performances between the standard and DSA planarization process will be compared. Figure 5 shows the top-view SEM images of the resulting 
dense and isolated patterns after BCP coating taken on two different wafers processed with the standard DSA process and the DSA planarization process, respectively. For the standard DSA process, the guiding patterns shape are not visible on the top-view SEM images of the most isolated guiding patterns; while the guiding patterns are still visible on the most dense patterns indicating a thinner BCP film. As already specified before, the BCP thickness inside guides varies with guiding pattern density. The same behavior is observed between the edge and the center of pattern fields: the BCP thickness is larger at the edge of pattern fields. In opposite, in the case of DSA planarization process, the guiding patterns are always visible regardless of the guiding pattern density. These SEM observations suggest an important difference between the two processes on how the guiding patterns are filled by the BCP. AFM measurements have been carried out at different pre-pattern pitches in order to determine the BCP thickness inside the guiding cavities. Figure 6 reports the percentage of guiding pattern filling by the BCP film determined by AFM and the HOY (Hole Open Yield) as a function of the guiding pattern pitch for both the DSA planarization process (in red) and the standard DSA process (in black). Without planarization, the BCP film thickness increases when the pitch of guiding patterns increases. Indeed, according to figure 6. (a), the BCP fills about $40 \%$ and $96 \%$ of the guiding cavity at $110 \mathrm{~nm}$ and $250 \mathrm{~nm}$ pitches, respectively, which confirms the BCP thickness variation with guiding pattern density when using the standard DSA graphoepitaxy process. Accordingly, the HOY (Hole Open Yield), defined as the ratio of valid shrunk holes to total inspected holes, ${ }^{[34]}$ reaches $100 \%$ for the most dense patterns but it drastically decreases down to zero when the guiding pattern pitch increases (figure 6. (b)). On the other hand, using the planarization process, the remaining BCP film thickness inside the cavity after etch-back is still constant over the pitch and thus the HOY is at $100 \%$ for all the guiding pattern densities. We have experimentally measured by AFM that constant BCP filling and 100\% 
HOY were achieved starting from a BCP over-thickness $(\mathrm{T})$ of $120 \mathrm{~nm}$. These results highlight the advantage of the planarization approach to overcome the issue of DSA density dependence. In addition, the planarization process allows us to avoid the issue of non-uniform filling between the center and the edge of the same pattern field, which was observed in the case of the standard DSA process flow. Finally, promising results have been obtained on contact doubling as reported in figure 5. These results indicate that the DSA planarization process can also be performed for contact multiplication applications.

\section{Conclusion}

We have developed a new DSA process flow called "DSA planarization" for contact-hole patterning by graphoepitaxy of PS- $b$-PMMA BCP. The process is based on guiding pattern overfilling by BCP film followed by a dry etch-back step. This process allows overcoming the issue of DSA defectivity dependence with pre-pattern density, previously encountered with the standard DSA process and considered as a major limitation for the DSA integration in IC manufacturing. Experimental results based on SEM imaging (top-view and cross-sectional) have shown that a vertical PMMA cylinder is perfectly obtained along the guiding pattern cavity after the BCP overfilling, whereas in-plane cylinders are observed at the top surface. Simulations based on a phase-field model have confirmed this experimental result. An etch-back step was then needed in order to reveal the buried BCP morphology and allow for the shrinkage of contacts. The etching step has been optimized and monitored using standard OES to accurately reach the in-depth vertical cylinder while keeping enough $\mathrm{BCP}$ thickness budget for subsequent etching transfer to underlayers. We have demonstrated by AFM measurements and SEM inspection that a uniform BCP thickness and 100\% HOY can be achieved at different pre-pattern densities (from $100 \mathrm{~nm}$ pitch up to $475 \mathrm{~nm}$ pitch) after the etch-back step. Thanks to the DSA planarization approach, a 
good control of the BCP thickness has been achieved on various pre-patterns densities (from dense to isolated) and morphologies (from shrink to multiplication) on the same processed wafer, which could prove very beneficial to the real integration case of IC devices. It is important to highlight that this new approach is also compatible for line and space patterning by DSA.

\section{Experimental Section}

DSA Materials: Polystyrene-b-poly(methyl methacrylate) (PS-b-PMMA) di-block copolymers with a respective weight ratio of 70:30 and a natural period $\left(\mathrm{L}_{0}\right)$ of $35 \mathrm{~nm}$ were used and referred as C35. PS-b-PMMA block copolymer was synthesized using sequential anionic polymerization, while random copolymers (PS-r-PMMA) containing different fractions of styrene and methylmethacrylate were synthesized through controlled radical polymerization. PS- $b$-PMMA and PS- $r$-PMMA were supplied by Arkema under the tradename Nanostrength EO $®$ C35 and Nanostrength EO® R respectively and used as received.

Process: The DSA process employed in this paper is based on the graphoepitaxy approach using organic hard mask guiding patterns. ${ }^{[3,34,35]}$ The guiding patterns were generated on $300 \mathrm{~mm}$ substrate wafers using a standard tri-layer stack composed of a resist, Si-ARC (Si containing antireflective coating) and SOC (spin on carbon). Various circular test structures with different dimensions, pitches for contact-hole shrink applications were designed in the electron sensitive resist by e-beam lithography on a VISTEC SB3054DW tool working at an accelerating voltage of $50 \mathrm{kV}$ and a current density of $20 \mathrm{~A} / \mathrm{cm}^{2}$. Then the SiARC and the SOC layers were etched subsequently by dry plasma processing in order to generate the organic guiding patterns cavities with straight profiles. Afterwards, the PS-r-PMMA solution is spin-coated on the guiding patterned wafers and then annealed at $250^{\circ} \mathrm{C}$ for 2 minutes to ensure the chemical grafting of a thin neutral layer. ${ }^{[35]}$ The surface is rinsed in PGMEA to remove the un-grafted materials. PS-b- 
PMMA solutions is spin-coated on the top of the PS-r-PMMA grafted neutral layer (NL) and annealed at $250^{\circ} \mathrm{C}$ to allow the self-assembly process to take place. The BCP film thickness was finely controlled by tuning the spin-coating speed and the initial solution concentration. For the DSA planarization process, the BCP over-thickness (T) on the top of cavity is set at $200 \mathrm{~nm}$. Afterwards, the BCP is self-assembled by thermal annealing. BCP film is annealed at $250^{\circ} \mathrm{C}$ for a period of $2 \mathrm{~min}$ and $5 \mathrm{~min}$ for respectively standard and DSA planarization process. After BCP's self-assembly inside guiding patterns, PMMA domains are selectively removed by wet development for the standard process while dry etch with $\mathrm{O}_{2}$-based chemistry is performed for the new DSA planarization process. All results presented in this work were obtained using the DSA Leti's 300mm pilot line. Coatings, bakes, PGMEA rinse and wet PMMA development were carried out on the DSA dedicated SOKUDO DUO track. Dry etching was performed on 300mm Inductive (ICP) Coupled Plasma Chambers from LAM VERSYS.

Characterization: Top-view and cross-sectional SEM images were obtained using Hitachi HCG4000 CD-SEM and Hitachi S5000, respectively. Mean CD and CDU determination are based on the analysis of top-view CD-SEM images taken on $300 \mathrm{~mm}$ wafers. The HOY, defined as the ratio of valid shrunk holes to total holes, is also determined at each guiding pattern based on topview SEM images inspection. Measurements were done over 100 contacts per chip on 70 chips on the wafer ( $~ 7000$ total inspected contacts per pattern) using the methodology described in Ref. 34 . BCP film thickness inside contact-holes was determined via step height measurements using an Insight3D AFM from Bruker.

Simulation: Phase field methodology has been chosen over other types of methods because of its reduced computational cost. To simulate the process we must take into account the overthickness brought by planarization, and so the simulation domain size is quite large (>10E5 DOF). 
Atomistic, coarse grained models or self-Consistent Field Theory are not relevant as the unnecessary level of precision induces huge computational cost. Papers from Wu \& Dzenis ${ }^{[36,37]}$ and Bosse $\mathrm{e}^{[38]}$ inspired the model's equations. The equations solved are: $\frac{\partial \varphi}{\partial t}=\Delta\left(\varphi^{3}-\varphi-\varepsilon^{2} \Delta \varphi\right)-\sigma\left(\varphi-\varphi_{0}\right)$ on $\Omega$

n. $\nabla \varphi=-\cos \theta$ on $\delta \Omega$

With parameters set to $\varepsilon=0.195, \sigma=1, \theta=\pi / 2$ at bottom and $\theta=0$ on walls, $\phi 0=-0.4$. To scale the geometry and parameters to experimental data we used scaling laws derived by Choksi ${ }^{[27,28]}$ that relate $\varepsilon$ and $\sigma$ to $\chi \mathrm{N}$ and $\mathrm{L}_{0}$. Finally, these equations have been solved using the Finite Element software Comsol.

\section{Acknowledgements}

The research work leading to these results has been performed in the frame of the collaborative program IDEAL with the support of all partners: ASML, ARKEMA, CEA-Leti, CNRS laboratories (LCPO and LTM), Mentor graphics, SOKUDO (Dainippon screen Mfg.), ST Microelectronics, TEL. The research leading to some of these results has received funding from the European Union's Horizon 2020 research and innovation program under grant agreement No 688072 IONS4SET. The authors thank Christine Tallaron, Maxime Argoud, Jessy Bustos, and Jonathan Pradelles for supporting with the SEM sample preparation and E-beam lithography. The authors are grateful to Sébastien Barnola for his helpful discussion and advices.

Received:

Revised:

Published online:

\section{References}

[1] H-C. Kim, S-M. Park, W. D. Hinsberg, Chemical Reviews 2010, 110 (1), 146-177

[2] J. Y. Cheng, D. P. Sanders, H. D. Truong, S. Harrer, A. Friz, S. Holmes, M. Colburn, W. D. Hinserg, ACS Nano 2010, 4(8), 4815-4823

[3] R. Tiron, A. Gharbi, M. Argoud, X. Chevalier, J. Belledent, P. Pimenta Barros, C. Navarro, G. Cunge, S. Barnola, L. Pain, M Asai, C Pieczulewski, Proc. of SPIE 2013, 8680-30 
[4] H. Tsai, J. W. Pitera, H. Miyazoe, S. Bangsaruntip, S. U. Engelmann, C-C. Liu, J. Y. Cheng, J. J. Bucchignano, D. P. Klaus, E. A. Joseph, D. P. Sanders, M. E. Colburn, M. A. Guillorn, ACS Nano 2014, 8 (5), 5227-5232

[5] H. Tsai, H. Miyazoe, J. B. Chang, J. Pitera, C-C. Liu, M. Brink, I. Lauer, J. Y. Cheng, S. Engelmann, J. Rozen, J. J. Bucchignano, D. P. Klaus, S. Dawes, L. Gignac, C. Breslin, E. A. Joseph, D. P. Sanders, M. E. Colburn, M. A. Guillorn, IEEE International Electron Devices Meeting 2014, San Francisco, CA, pp. 32.1.1-32.1.4

[6] X-Y. Bao, H. Yi, C. Bencher, L-W. Chang, H. Dai, Y. Chen, P-T. Joseph Chen; H-S. Philip Wong, IEEE International Electron Devices Meeting 2011, pp. 1-4

[7] H. Yi, X.-Y. Bao, J. Zhang, C. Bencher, L-W. Chang, X. Chen, R. Tiberio, J. Conway, H. Dai, Y. Chen, S. Mitra, H-S. Philip Wong, Adv. Mat. 2012, 24, 3170-3114

[8] C-C. Liu, E. Han, M. S. Onses, C. J. Thode, S. Ji, P. Gopalan, P. F. Nealey, Macromolecules 2011, 44(7), 1876-1885

[9] R. Ruiz, H. Kang, F. A. Detcheverry, E. Dobisz, D. S. Kercher, T. R. Albrecht, J. J. De Pablo, P. F. Nealey, Science 2008, Vol 321, Issue 5891

[10] L. Oria, A. R. De Luzuriaga, X. Chevalier, J. A. Alduncin, D. Mecerreyes, R. Tiron, S. Gaugiran, F. Perez-Murano, Proc. of SPIE. 2011, Vol. 7970

[11] Y. Seino, H. Yonemitsu, H. Sato, M. Kanno, H. Kato, K. Kobayashi, A. Kawanishi, T. Azuma, M. Muramatsu, S. Nagahara, T. Kitano, T. Toshima, Journal of Micro/Nanolithograhy MEMS, and MOEMS 2013, 12(3), 033011

[12] P. Pimenta Barros, S. Barnola, A. Gharbi, M. Argoud, I. Servin, R. Tiron, X. Chevalier, C. Navarro, C. Nicolet, C. Lapeyre, C. Monget, E. Martinez, Proc. Of SPIE 2014, 9054, Advanced Etch Technology for Nanopatterning III, 90540G 
[13] R. Gronheid, J. Bekaert, V.-K. M. Kuppuswamy, N. Vandenbroeck, J. Doise, Y. Cao, G.

Lin, S. Sayan, D. Parnell, M. Somervell, Proc. Of SPIE 2014, 9051, Advances in Patterning Materials and Processes XXXI, 90510I

[14] J. Bekaert, J. Doise, V.-K. M. Kuppuswamy, R. Gronheid, B.T. Chan, G. Vandenberghe, Y. Cao, Y. Her, Photomask BACUS news 2014, Vol. 30, issue 8

[15] J. Doise, J. Bekaert, B. Chan, S. Hong, G. Lin, R. Gronheid, Journal of Micro/Nanolithograhy MEMS, and MOEMS 2016, 15(3), 031603

[16] A. Latypov, T. H. Coskun, G. Garner, M. Preil, G. Schmid, J. Xu, Y. Zou, Proc. Of SPIE 2014, 9049, Alternative Lithographic Technologies VI, 904908

[17] H. Yi, J. Bekaert, R. Gronheid, G. Vandenberghe, K. Nafus, H.-S. P. Wong, Proc. Of SPIE 2015, 9423, Alternative Lithographic Technologies VII, 94231F

[18] J. Bekaert, J. Doise, R. Gronheid, J. Ryckaert, G. Vandenberghe, G. Fenger, Y. J. Her, Y. Cao, Proc. Of SPIE 2015, 9658, Photomask Japan, Photomask and Next-Generation Lithography Mask Technology XXII, 965804

[19] P. Pimenta Barros, A. Gharbi, R. Tiron, X. Chevalier (CEA-Leti, Arkema), Patent US 20160077439 A1, 2015

[20] P. Pimenta Barros, A. Gharbi, A. Sarrazin, R. Tiron, N. Posseme, S. Barnola, S. Bos, C. Tallaron, G. Claveau, X. Chevalier, M. Argoud, I. servin, C. Navarro, C. Nicolet, C. Lapeyre, C. Monget, Proc. Of SPIE 2015, 9428, Advanced Etch Technology for Nanopatterning IV, 94280D [21] R. Tiron, A. Gharbi, P. Pimenta Barros, S. Bouanani, C. Lapeyre, S. Bos, A. Fouquet, J. Hazart, X. Chevalier, M. Argoud, G. Chamiot-Maitral, S. Barnola, C. Monget, V. Farys, S. BerardBergery, L. Perraud, C. Navarro, C. Nicolet, G. Hadziioannou, G. Fleury, Proc. Of SPIE 2015, 9423, Alternative Lithographic Technologies VII, 942317 
[22] L. E. Stillwagon, R. G. Larson, G. N. Taylor, Journal of the Electrochemical Society 1987, 134(8), 2030-2037

[23] A. G. Sunil, K. G. Rakesh, Ind. Eng. Chem. Res 1998, 37, 2223-2227

[24] E. Pargon, K. Menguelti, M. Martin, A. Bazin, O. Chaix-Pluchery, C. Sourd, S. Derrough, T. Lill, O. Joubert, Journal of applied physics 2009, 105, 094902

[25] S. Yoshimura, Y. Tsukazaki, M. Kiuchi, S. Sugimoto, S. Hamaguchi, J. Phys. D: Appl. Phys 2012, 45505201 (10pp)

[26] T. Ohta, K. Kawasaki, Macromolecules 1986, 19, 2621-2632

[27] R. Choksi, M. A. Peletier, J. F. Williams, SIAM Journal on Applied Mathematics 2009, 69, $1712-1738$

[28] R. Choksi, X. Ren, Journal of Statistical Physics 2003, 113, 151-176

[29] J. W. Coburn, M. Chen, J. Appl. Phys. 1980, 51.6, 3134-3136

[30] C. A. Ross, K. K. Berggren, J Y. Cheng, Y. S. Jung, J-B Chang, Adv. Mater. 2014, 26, 4386-4396.

[31] A. Tavakkoli. K. G., K. W. Gotrik, A. F. Hannon, A. Alexander-Katz, C-A. Ross, K. K. Berggren, Science 2012, vol. 336, Issue 6086, pp 1294-1298

[32] G-W Yang, G-P Wu, X chen, S. Xiong, C. G. Arges, S. Ji, P. F. nealey, X-B. Lu, D. J. Darensbourg, Z-K. Xu, Nano Lett. 2017, 17, 1233-1239

[33] T. Seshimo, R. Maeda, R. Odashima, Y. takenaka, D. Kawana, K. Ohmori, T. Hayakawa, Sci. Rep. 2016, 6, 19481

[34] A. Gharbi, R. Tiron, M. Argoud, X. Chevalier, P. Pimenta Barros, C. Nicolet, C. Navarro, Journal of Micro/Nanolithograhy MEMS, and MOEMS 2015, 14(2), 023508 
[35] I. Servin, R. Tiron, A. Gharbi, M. Argoud, K. Jullian, G. Chamiot-Maitral, P. Pimenta Barros, X. Chevalier, J. Belledent, X. Bossy, S. Moulis, C. Navarro, G. Cunge, S. Barnola, M. Asai, C. Pieczulewski, Japanese Journal of Applied Physics 2014, 53, 06JC05

[36] X.-F. Wu, Y. A. Dzenis, The Journal of Chemical Physics 2006, 125, 174707

[37] X.-F. Wu, Y. A. Dzenis, Physical Review 2008, E 77

[38] A. W. Bosse, Macromolecular Theory and Simulations 2010, 19, 399-406 
(a) Standard graphoepitaxy process flow

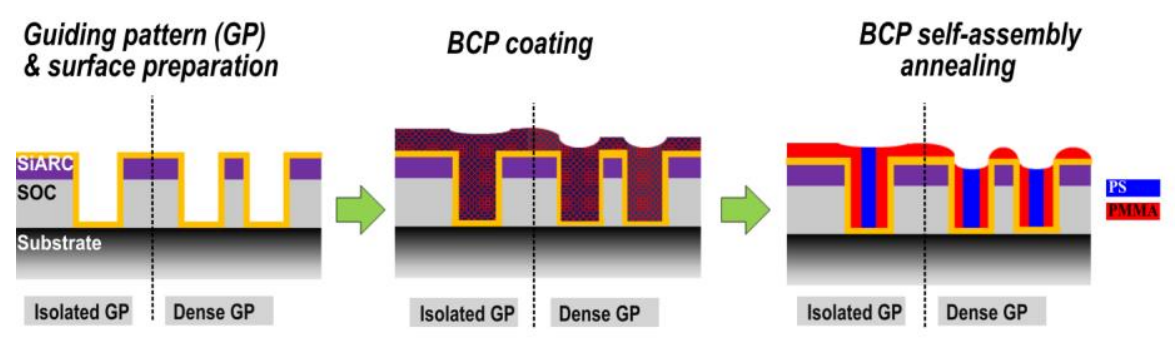

(b) DSA planarization process flow

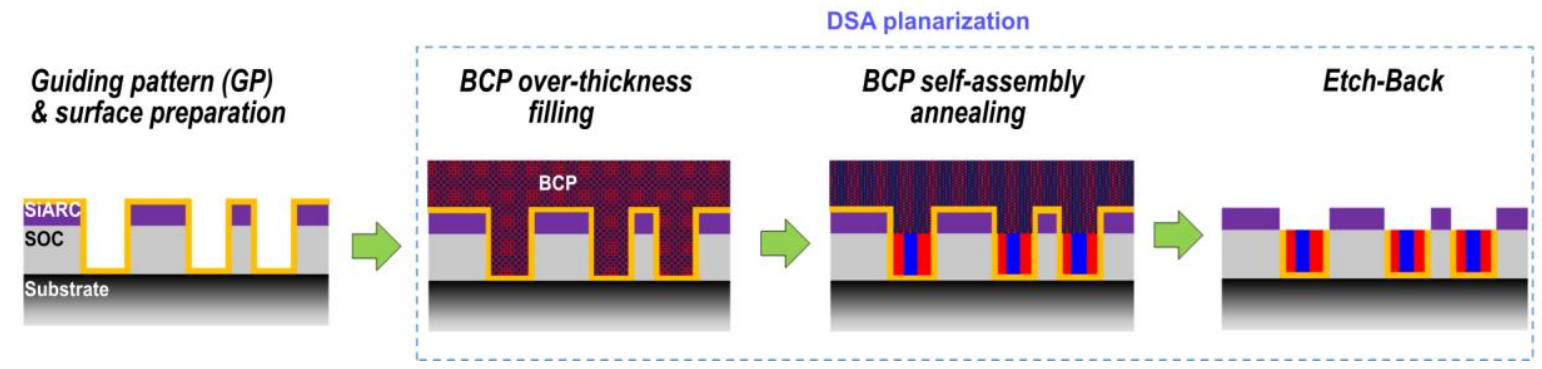

Figure 1. Schematic representation of isolated and dense guiding patterns filling by BCP film when using (a) standard graphoepitaxy and (b) DSA planarization process flows 
(a) $2 D$ Side view as a function of $B C P$ over-thickness (T)

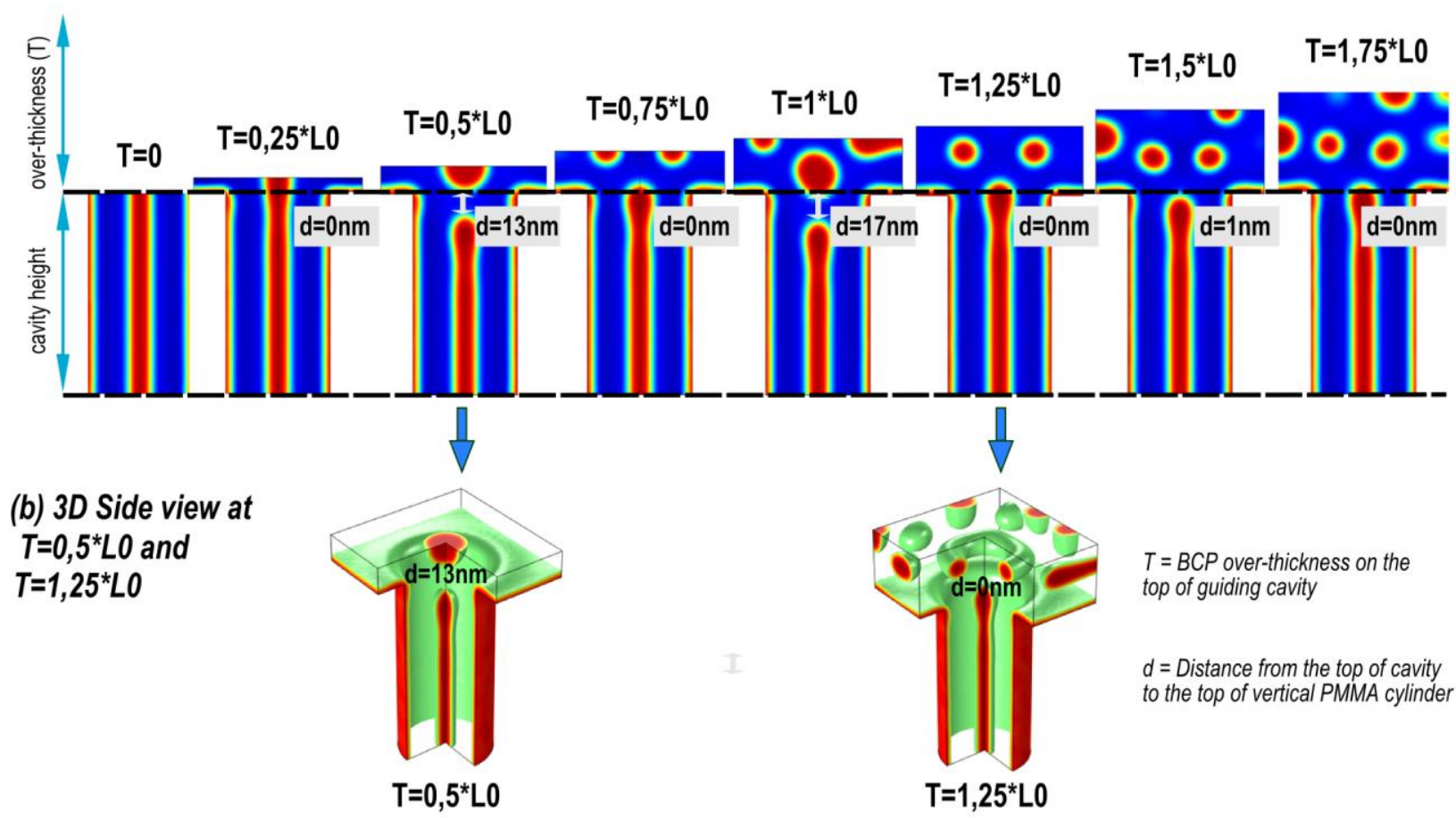

Figure 2. (a) side view of the simulated guiding patterns as a function of the BCP overthickness (T). PMMA phase is in red, PS phase in blue. (b) 3D cut-view of simulation, the PMMA phase is in red. Two different conformations are emerging depending on the BCP over-thickness: for $\mathrm{T}=0,5^{*} \mathrm{~L}_{0}$, a semi sphere is formed on top of the cavity "pushing" the cylinder into the cavity, while for $\mathrm{T}=1,25 * \mathrm{~L}_{0}$, a torus is formed above the cavity without impacting the vertical cylinder inside cavity that reaches the top 


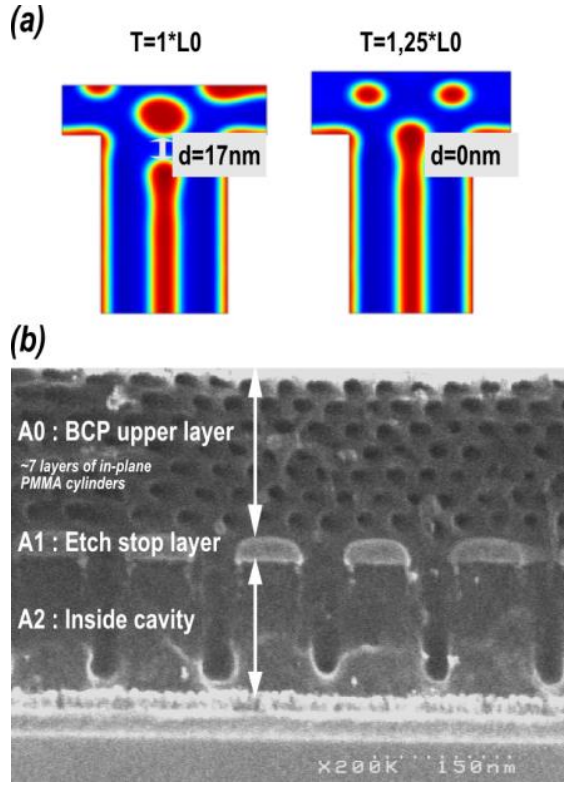

Figure 3. (a) the two main conformations of simulation results and (b) SEM cross-section of guides overfilled by BCP film

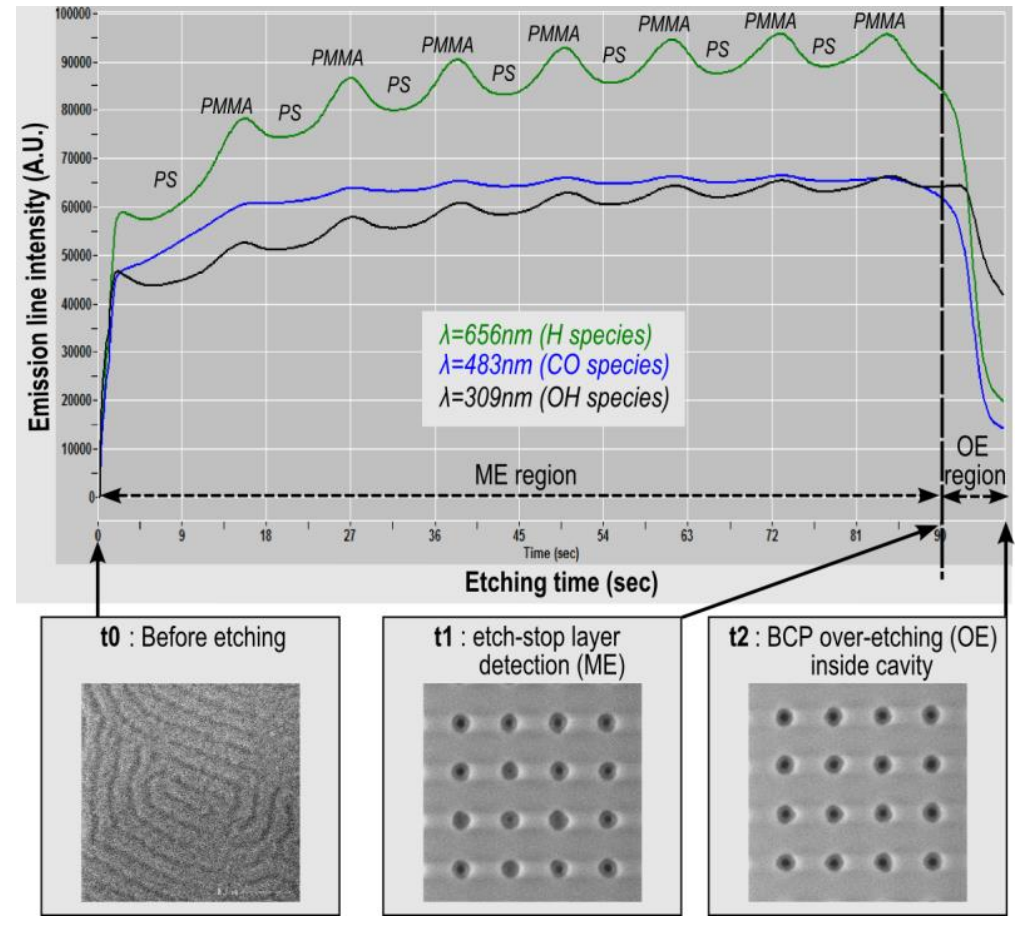

Figure 4. In-situ plasma monitoring using Optical Emission Spectrocopy: $656 \mathrm{~nm}, 483 \mathrm{~nm}$ and $309 \mathrm{~nm}$ wavelength intensity as a function of etching time (curves). And top-view SEM image (inset) taken at different etching time $\left(\mathrm{t}_{0}\right.$, $\left.t_{1}, t_{2}\right)$ 


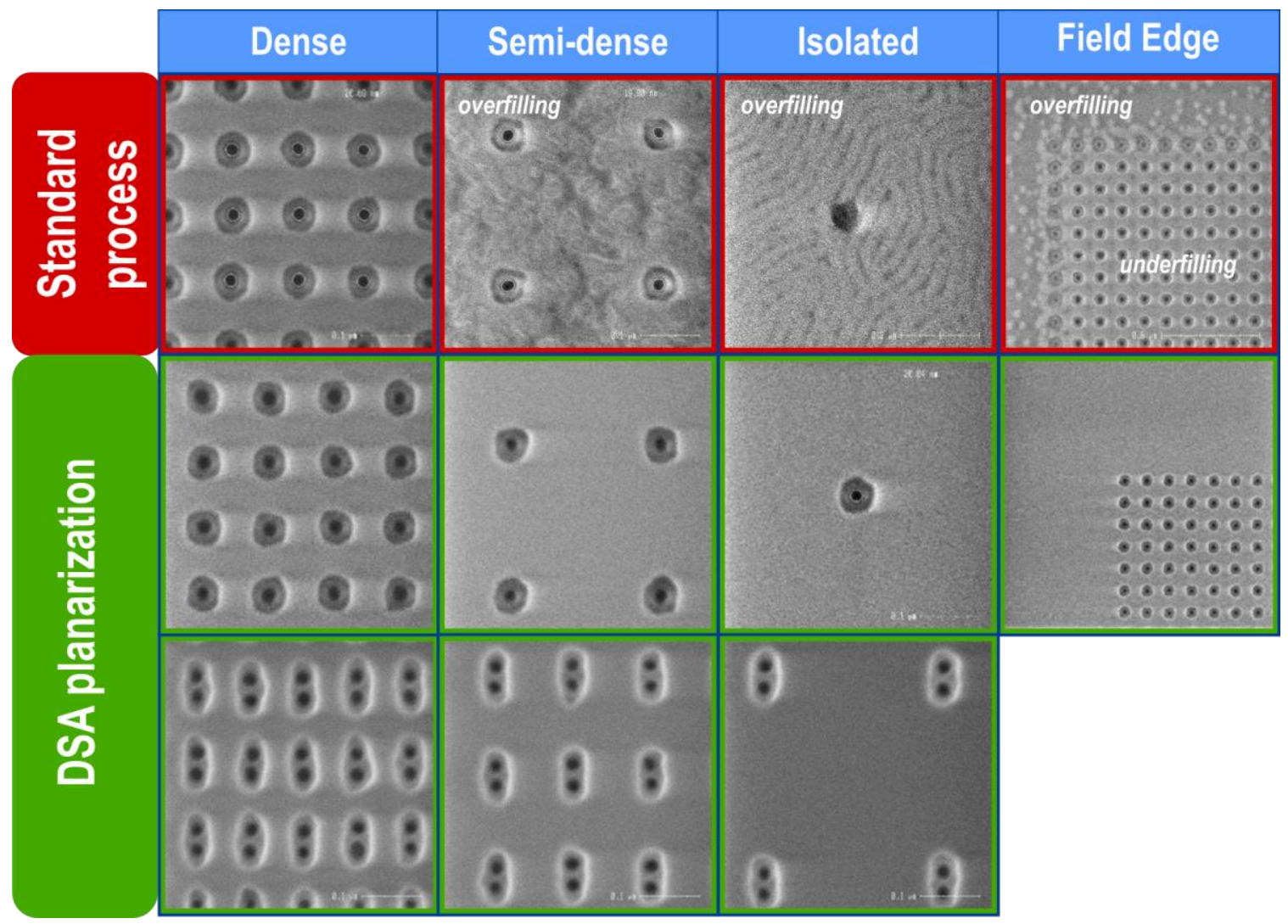

Figure 5. Top-view SEM images of DSA patterns at different guiding prepattern densities for standard and DSA planarization process flows. Contact-hole shrink and doubling can be performed on the same processed wafer when using the DSA planarization process 

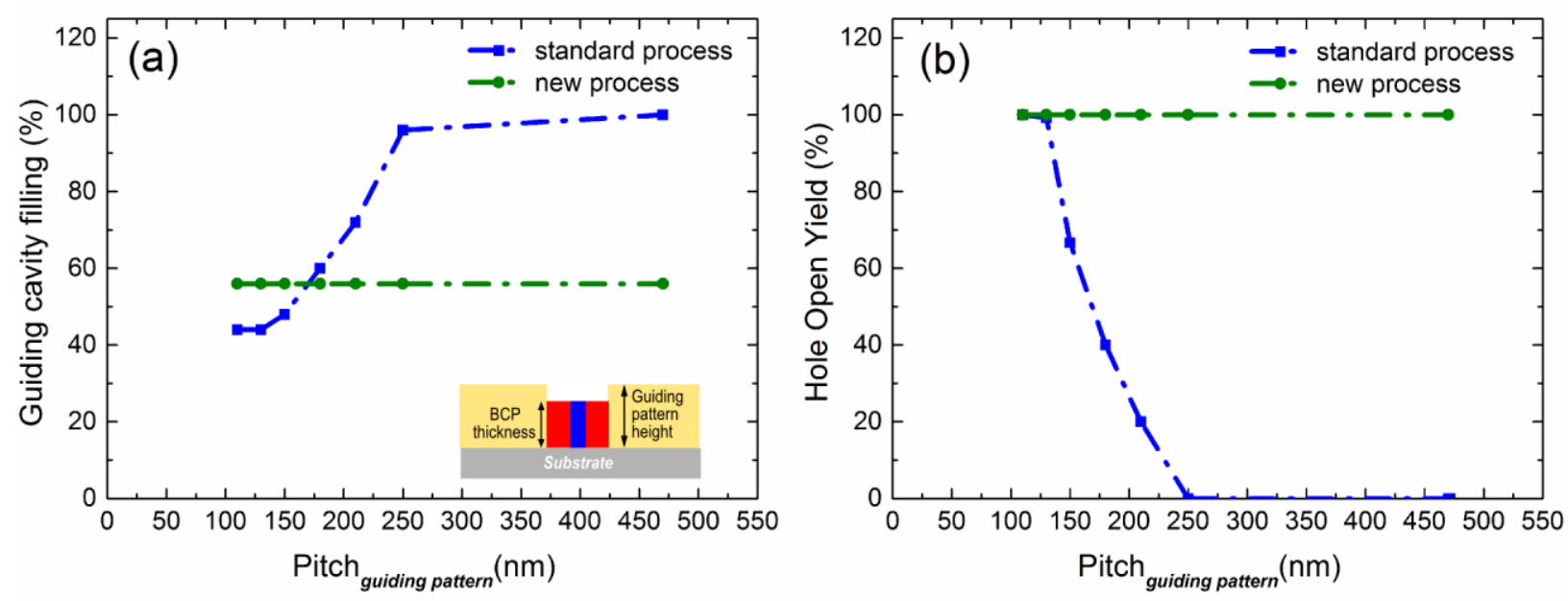

Figure 6. Guiding pattern filling (a) and Hole Open Yield (b) as a function of guiding pitch for standard (squares) and new (round) process, i.e. with and without DSA planarization. When the guiding cavity was overfilled, the guiding cavity filling was fixed at $100 \%$. 
Uniform block copolymer (BCP) thickness over template density is achieved using a new and simple graphoepitaxy process flow called "DSA planarization". Simulations and SEM characterizations of BCP self-assembly overfilling the guiding patterns confirm the viability of this approach. Experimental results demonstrate the enhancement of DSA performances when using the DSA planarization flow.

Keywords: Directed Self-Assembly (DSA), block copolymer, graphoepitaxy, pattern density, planarization

P. Pimenta Barros*, A. Gharbi, A. Fouquet, S. Bos, J. Hazart, F. Delachat, X. Chevalier, I. Cayrefourcq, L. Pain, R. Tiron

Balancing Block Copolymer Thickness over Template Density in Graphoepitaxy Approach

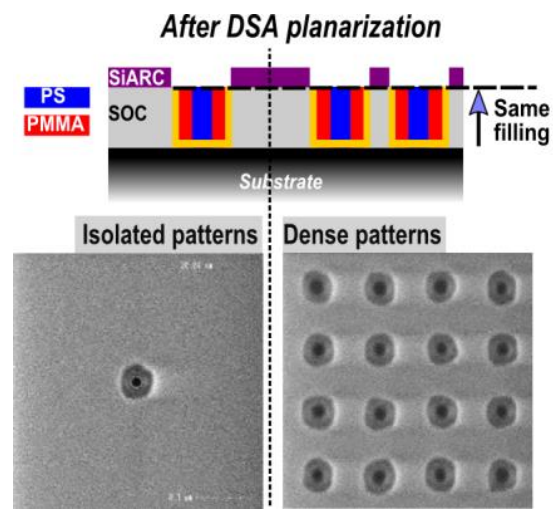

